MEDICAL RESEARCH

\section{Oncogenesis Debated}

from a Correspondent

IN the lecture theatre of the British Museum (Natural History), some years ago, was held a symposium on aggression at which the audience was told that ritualization is a device adopted by many living species for keeping arguments within reasonable bounds. At the same venue on January 17 , an audience was treated to an example of ritualized aggression between Dr J. F. Watkins (Sir William Dunn School of Pathology, Oxford) and Professor Sir David Smithers (Royal Marsden Hospital, London) during the second meeting in the series on principles of experimental and clinical oncology organized by the Institute of Cancer Research (see Nature, 235, 129 ; 1972). Sir Oliver Scott was the referee. The aim was to dissect the roles of cell and environment in oncogenesis. Dr Watkins felt that understanding at the molecular level could form the basis of a cure. His presentation rested on an analytical approach to the nature of malignant change largely carried out in vitro. Sir David, with thirty-six years of clinical experience, considered that cancer is an organizational defect.

Dr Watkins first distinguished between growth control and topological control. Different degrees of each were named, by reference to the terminology of bacterial genetics, stringent and relapsed. For full malignancy, Dr Watkins envisaged that topological and growth control mechanisms are relapsed. Benign tumours were thought to have lost growth control but to be contained by their surroundings and thus still sensitive to topological controls.

The early in vitro studies by Sanford and Earle had established that a change to a malignant condition could occur in vitro though, of course, the change could only be proved by implantation to a living animal. But the defect of these studies and later work using chemical carcinogens was the uncertain time lapse between the application of the stimulus to change and the change itself. Dr Watkins felt that carcinogenesis by viruses in vitro did not suffer from this drawback and he devoted the rest of his talk to this topic. He excluded RNA viruses from consideration. Part of the difficulty, Dr Watkins said, was that it was impossible to establish a perfect correlation between malignant change and some feature of cells in vitro such as morphological transformation, loss of contact inhibition or agglutinability by the wheat germ lectin. For example, it seemed that reversion to a non-malignant condition could occur in tissue culture with
SV 40 transformed cells of a mouse embryo strain. Although contact inhibition was regained, hovever, the capacity to form a tumour in an appropriate host was more sporadic. Perhaps the delineation of what is a suitable host is the weak link here.

Dr Watkins turned to consider whether malignancy could be attributed to an oncogene (following Huebner). In outline, he said it was an easy question to answer-simply hybridize malignant and non-malignant cells and determine whether the resulting heterokaryons could grow in an immunologically suppressed host. The story is complicated, however, by the finding that the result obtained depends on the number of chromosomes lost from the hybrid cells. The results could, overall, be said to support either a deletion or recessive mutational hypothesis, but until there developed some means for cloning hybrid cells without loss of chromosomal material no definitive answers would emerge. Dr Watkins final con. clusion was that one should learn about the molecular basis of malignant change but that the learning would involve a slow collection with "heat and acrimony" of many apparently disconnected facts.

As a clinician, Sir David Smithers had collected a different set of criteria for malignant cell populations. The things that impressed him were the many predisposing factors, the long latency, multicentric origins, increasing autonomy, that site frequency was related to the growth characteristics of a tissue or organ and that tumour cell populations were heterogeneous. Of secondary interest were the tendency to metaplasia, conditional persistence, hormone dependency, maturation and spontaneous regression. Of these factors, he felt hormone dependence was the most significant discovery. Sir David, quoting his elders, tried to establish that cancer should be thought of as a distortion of normal tissue rather than a distinct qualitative change. Furthermore, he insisted that cancer should be described in relation to the whole organism.

\section{NEONATES

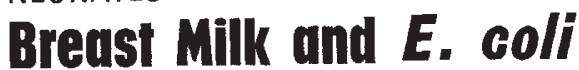

from a Correspondent GASTROENTERITIS is often considered to be a mild disorder, but in babies in the first few weeks of life it can be lethal. Paediatricians are thus most interested in the observation that breast-fed babies are less at risk. The greater problems of hygiene involved in bottle-feeding a baby seem not to be the whole story, and it is important to know the factor in breast milk that increases the resistance to gastroenteritis.
It is the multiplication of bacteria, most usually certain forms of Escherichia coli, that can exist harmlessly in the bowel in small numbers that causes gastroenteritis, and this increase is less common when babies are breast-fed. $E$. coli reproduces more slowly in an acid environment, such as is produced in the bowel by a harmless bacteria Lactobacillus bifidus; these bacteria are more plentiful in the intestines of breast-fed babies.

But it now seems that this may not be the only mechanism involved in the disorder. J. J. Bullen, H. J. Rogers of the National Institute for Medical Research, Mill Hill, and L. Leigh of the Rowett Research Institute, Aberdeen, have shown that human milk can have a specific inhibitory effect on $E$. coli. They attribute this effect to the action of an iron-binding protein-lactoferrin, and possibly the related substance transferrin-in combination with a specific antibody to $E$. coli which stops its multiplication (Brit. Med. J., 1, 69; 1972).

In experiments in vitro under conditions of controlled $p \mathrm{H}$, Bullen, Rogers and Leigh found that lactoferrin alone added to $E$. coli reduced the viable count to one fiftieth of the control, and the addition of $E$. coli antiserum slightly reduced the viable count. Used together, however, there was a pronounced effect in which the viable count was reduced to about one threethousandth of the control. Human milk gave comparable results in the same series of experiments. It seems to be the iron-binding properties of lactoferrin which are significant, for Bullen, Rogers and Leigh found that the saturation of lactoferrin with iron negated the inhibitory effect. Why this should be so is not at all clear.

So how are the two possible mechanisms for inhibiting $E$. coli related? Bullen, Rogers and Leigh investigated this point by experiments in guineapigs, whose milk for these purposes resembles human milk. What seems to happen is that the inhibitory effect of lactoferrin with specific antisera operates to keep down $E$. coli for the first few days after birth until the bowel has been well colonized with Lactobacilli.

By clarifying the mechanisms by which breast-feeding confers resistance to gastroenteritis, this work will give further substance to the argument that breast-feeding is in general better than artificial feeding. Cow's milk contains less lactoferrin than human milk, and although the amount of transferrin is greater, it may well be denatured by the processing that makes the milk digestible. Furthermore, the addition of iron to cow's milk to bring the iron content up to the level found in human milk probably saturates the remaining lactoferrin and transferrin. 